\title{
Remanence breakdown in granular alloys at magnetic percolation
}

\author{
X. Batlle, V. Franco, and A. Labarta ${ }^{a}$ \\ Departmento Física Fonamental, Facultat de Física, Universitat de Barcelona, Av. Diagonal 647, \\ 08028 Barcelona, Spain \\ K. O'Grady \\ Magnetic Materials Research Group, SEES, UCNW Bangor, Gwynedd, LL57 1UT, United Kingdom
}

(Received 9 August 1999; accepted for publication 20 April 2000)

\begin{abstract}
Microstructural effects on the magnetic behavior of rf-sputtered $\mathrm{CoFe}-\mathrm{AgCu}$ granular alloys are examined through the study of the in-plane remanence-to-saturation magnetization ratio, $M_{r} / M_{s}$, as a function of temperature, ferromagnetic volume content, $x_{v}$, and annealing temperature. At low ferromagnetic contents $\left(x_{v} \leqslant 0.25\right)$, the $M_{r} M_{s}$ ratio in as-deposited samples tends towards 0.5 at low temperature, as expected from the uniaxial perpendicular anisotropy displayed by all samples, which is magnetoelastic in nature and arises from the axial distortion of the CoFe face-centered-cubic cells. In as-deposited samples with $x_{v}>0.25$ (well below the volume percolation threshold, $x_{p}$ $\sim 0.5-0.55$ ), a collective magnetic behavior develops due to magnetic correlations among particles. Consequently, a domain structure perpendicular to the film plane appears, which results in a remanence breakdown: $M_{r} / M_{s}$ is about 0.2 . Besides, magnetic correlations prevents the thermal decay of $M_{r} / M_{s}$, which is almost constant between 5 and $300 \mathrm{~K}$, even for ferromagnetic particles no more than $3 \mathrm{~nm}$ in size. The axial distortion disappears with annealing, the cubic symmetry is recovered, the out-of-plane magnetic structure is lost and, therefore, the $M_{r} / M_{s}$ ratio at low temperature tends towards 0.8 for highly annealed samples. Consequently, the magnetic properties of granular alloys depend on the interplay between anisotropy, exchange, and dipolar interactions, which in turn depend crucially on the microstructure. (C) 2000 American Institute of Physics.
\end{abstract}

[S0021-8979(00)00115-8]

\section{INTRODUCTION}

Magnetic granular alloys, consisting of a distribution of fine magnetic particles embedded in a nonmagnetic metallic matrix, have generated great interest due to their technological applications and their new challenging magnetic and transport properties, such as giant magnetoresistance. ${ }^{1}$ Technological requirements make the role of interparticle interactions increasingly important. ${ }^{2,3}$ In granular structures, exchange coupling is developed through the conducting matrix, while dipolar interactions are intrinsic in dispersions of fine magnetic particles.

The temperature decay of remanence is found to provide useful information about the energy barrier distribution, ${ }^{4}$ which can be used to examine the effects of interactions when compared to the low-field susceptibility and time dependence of the thermoremanence. ${ }^{5}$ At $T=0 \mathrm{~K}$, the StonerWohlfarth model ${ }^{6}$ predicts that the remanence-to-saturation magnetization ratio, $M_{r} / M_{s}$, is 0.5 for a random distribution of noninteracting uniaxial single domain particles with coherent rotation of the total magnetization. However, deviations from this ideal value are observed in real systems due to interparticle interactions and textured growth. The effect of dipolar interactions on $M_{r} / M_{s}$ is strongly dependent on both their strength and local particle distribution, and they may either enhance or reduce remanence. ${ }^{7-11}$ For example, a

a) Author to whom correspondence should be addressed; electronic mail: amilcar@ffn.ub.es particular topological arrangement of the particles (e.g., linear chain of regular particles) may lead to a nose-to-tail ordering of the magnetic moments and thus to an enhancement of the magnetization. ${ }^{12}$ However, experimental remanence at high concentration is usually found to decrease in a random distribution of fine particles due to dipolar interactions, ${ }^{9,10}$ in agreement with Monte Carlo simulations. ${ }^{7}$

Similarly, the effect of exchange interactions is difficult to predict since it also may either enhance or reduce magnetization. It has been observed that as the ferromagnetic (FM) content is increased and magnetic particles become closer, the increase in exchange coupling enhances remanence. ${ }^{8,13,14}$ However, weak exchange coupling may also enhance the formation of flux-closure loops, leading to a reduced remanent magnetization. Remanence enhancement has been widely studied in systems with coexisting soft and hard magnetic phases, and $M_{r} / M_{s}$ values up to 0.964 have been reported in recording media. ${ }^{15}$

$M_{r} / M_{s}$ values smaller than 0.5 have been reported in uniaxial systems, ${ }^{16-19}$ which has been attributed to quantum relaxation, ${ }^{16}$ interaction effects, ${ }^{17,18}$ or $M_{s}$ being overestimated due to surface and dilution effects. ${ }^{19}$ An increase in the blocking temperature with particle concentration has also been found due to dipolar interactions. ${ }^{3,9,19}$ Magnetic interactions also cause a slower thermal decay of the remanence.

In this article we study the $M_{r} / M_{s}$ ratio for $\mathrm{CoFe}-\mathrm{AgCu}$ granular alloys with FM volume contents, $x_{v}$, ranging from 0.10 to 0.45 . In as-deposited samples, the mean $\mathrm{CoFe}$ particle size and $\mathrm{CoFe}$ alloying to the matrix are irrespective of 
$x_{v}$, which means we can study: (i) the effect of magnetic interactions as a function of interparticle distance and (ii) the way in which magnetic correlations cause a remanence breakdown at magnetic percolation. The physical and magnetic microstructural changes with annealing are also monitored by remanence measurements.

\section{EXPERIMENT}

$\mathrm{CoFe}-\mathrm{AgCu}$ films 200-300 thick were rf sputtered onto glass substrates. $\mathrm{Fe}$ and $\mathrm{Cu}$ were added to the widely studied $\mathrm{Co}-\mathrm{Ag}$ system for a number of reasons: $\mathrm{Fe}$ was added to $\mathrm{Co}$ in order to increase the magnetic moment and, consequently, the magnetoresistive effect, ${ }^{20}$ while a small amount of $\mathrm{Cu}$ (about $3 \%$ by volume) was added to increase the $\mathrm{Co}-\mathrm{Ag}$ immiscibility. ${ }^{21} \mathrm{Cu}$ is immiscible in both $\mathrm{Co}$ and $\mathrm{Ag}$, which leads to a granular system even for as-deposited samples, obviating substrate heating or postdeposition annealing, but not avoiding a certain degree of $\mathrm{CoFe} \mathrm{AgCu}$ alloying. Postdeposition phase segregation was promoted by rapidly annealing $(0.1 \mathrm{~s})$ at $600,650,700$, and $750{ }^{\circ} \mathrm{C}$. A detailed description of the experimental procedure and structural characterization is given elsewhere. ${ }^{21}$ Parallel and perpendicular hysteresis loops were measured with a vibrating sample magnetometer with fields up to $12 \mathrm{kOe}$, within 5 and $300 \mathrm{~K}$. In-plane remanence curves (isothermal remanent magnetization and dc demagnetizing curves) were also measured at $5 \mathrm{~K}$. The field sweeping rate was kept constant and all samples were carefully ac demagnetized before any experiments were performed in order to avoid magnetic-history dependence. Susceptibility measurements at low field were performed with a superconducting quantum interference device magnetometer, in the in-plane geometry.

\section{RESULTS AND DISCUSSION}

\section{A. Microstructure and magnetic anisotropy}

Structural characterization confirms the granular nature of the samples for all the concentrations studied. ${ }^{21}$ Both $\mathrm{CoFe}$ and $\mathrm{Ag}$ crystals displayed a face-centered-cubic (fcc) structure with the $\langle 111\rangle$ direction textured perpendicular to the film plane, with an angular dispersion for the CoFe particles of about $30^{\circ}$ in as-deposited samples and about $10^{\circ}$ in samples annealed at $750{ }^{\circ} \mathrm{C}$. Structural data also showed a rhombohedral distortion of the cubic cell in all as-deposited samples: The $\langle 111\rangle$ direction is in compression, while the

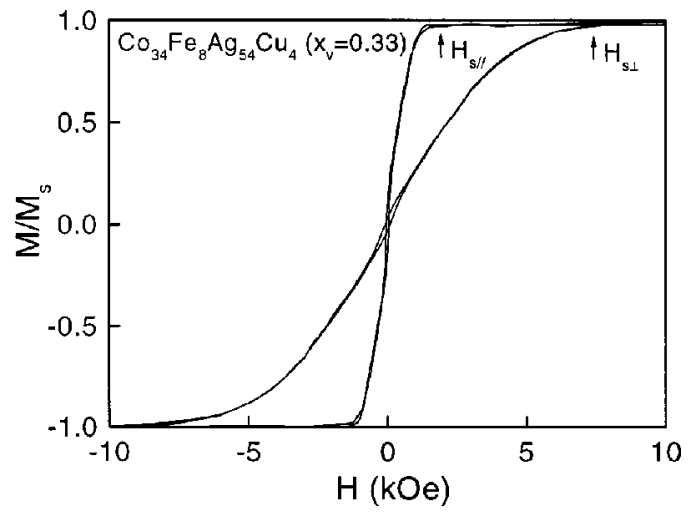

FIG. 1. Normalized parallel and perpendicular hysteresis loops at $300 \mathrm{~K}$ for a $\mathrm{Co}_{34} \mathrm{Fe}_{8} \mathrm{Ag}_{54} \mathrm{Cu}_{4}\left(x_{v}=0.33\right)$ as-deposited sample.

directions in the film plane are in tension, giving rise to a perpendicular uniaxial magnetic anisotropy along the textured $\langle 111\rangle$ direction. ${ }^{21}$ This axial distortion is associated with the residual stresses produced during deposition by differences in the thermal expansion coefficients of the substrate and the film. ${ }^{22-24}$

The value of the perpendicular uniaxial anisotropy may be evaluated by comparing parallel and perpendicular hysteresis loops (Fig. 1): ${ }^{25}$ The angle $\theta$ of the resulting anisotropy $K$ with respect to the film normal may be found using the expressions, $H_{s \|}=2 K \cos ^{2} \theta / M_{s}$ and $H_{s \|}+H_{s \perp}=4 \pi M_{s}$ $+2 K / M_{s}$, where $H_{s \|}$ and $H_{s \perp}$ are the parallel and perpendicular saturation fields, respectively, and $M_{s}$ is the saturation magnetization. The values of $M_{s}, H_{s \|}, H_{s \perp}, K$, and $\theta$ obtained are listed in Table I. Values for $K$ within $0.30-0.40 \times 10^{6} \mathrm{erg} / \mathrm{cm}^{3}$ and $\theta=0$ for all as-deposited samples have been found, in agreement with the magnetoelastic energy calculation from the axial distortion. ${ }^{21}$ Similar results have been observed in $\mathrm{CoFe}-\mathrm{Cu}$ granular films, ${ }^{26}$ though $K$ values were smaller $\left(\sim 0.2 \times 10^{6} \mathrm{erg} / \mathrm{cm}^{3}\right)$, as a consequence of a lower degree of texture with respect to the $\mathrm{CoFe}-\mathrm{AgCu}$ films.

The mean size of CoFe particles is about $3 \mathrm{~nm}$ for all as-deposited samples. This mean size irrespective of $x_{v}$ may be understood bearing in mind that the process of forming particles at $x_{v}$ below the percolation threshold $\left(x_{p}\right.$ $\sim 0.5-0.55)$ is a segregation-nucleation diffusion and growth process, in such a way that by stopping it at the earlier steps, the final size of the particles only depends on

TABLE I. Angle of the magnetic anisotropy with respect to the film normal $(\theta)$ and anisotropy constant $(K)$ for various $x_{v}$ and annealing temperatures, for $\mathrm{CoFe}-\mathrm{AgCu}$ alloys at $300 \mathrm{~K} . M_{s}$ is the saturation magnetization. $H_{s \|}$ and $H_{s \perp}$ are the parallel and perpendicular saturation fields, respectively. The thickness of the films are as follows: $260 \mathrm{~nm}$ for $x_{v}=0.15,240 \mathrm{~nm}$ for $x_{v}=0.19,355 \mathrm{~nm}$ for $x_{v}=0.25$, and $210 \mathrm{~nm}$ for $x_{v}=0.33$.

\begin{tabular}{llcccr}
\hline \hline \multicolumn{1}{c}{ Sample } & $x_{v}$ & $M_{s}\left(\mathrm{emu} / \mathrm{cm}^{3}\right)$ & $H_{s \|}(\mathrm{Oe})$ & $H_{s \perp}(\mathrm{Oe})$ & $K\left(10^{6} \mathrm{erg} / \mathrm{cm}^{3}\right)$ \\
\hline $\mathrm{Co}_{15} \mathrm{Fe}_{5} \mathrm{Ag}_{79} \mathrm{Cu}_{4}$ as-deposited & 0.15 & 254 & 2000 & 3400 & $0.28 \pm 0.05$ \\
$\mathrm{Co}_{18} \mathrm{Fe}_{8} \mathrm{Ag}_{70} \mathrm{Cu}_{4}$ as-deposited & 0.19 & 327 & 2000 & 4200 & $0.33 \pm 0.05$ \\
$\mathrm{Co}_{20} \mathrm{Fe}_{12} \mathrm{Ag}_{60} \mathrm{Cu}_{8}$ as-deposited & 0.25 & 422 & 1900 & 5300 & $0.39 \pm 0.05$ \\
$\mathrm{Co}_{20} \mathrm{Fe}_{12} \mathrm{Ag}_{60} \mathrm{Cu}_{8} 650^{\circ} \mathrm{C}$ & 0.25 & 440 & 2900 & 8200 & $0.94 \pm 0.10$ \\
$\mathrm{Co}_{34} \mathrm{Fe}_{8} \mathrm{Ag}_{54} \mathrm{Cu}_{4}$ as-deposited & 0.33 & 539 & 1650 & 6800 & $49 \pm 7$ \\
$\mathrm{Co}_{34} \mathrm{Fe}_{8} \mathrm{Ag}_{54} \mathrm{Cu}_{4} 450^{\circ} \mathrm{C}$ & 0.33 & 563 & 1850 & 7360 & $0.38 \pm 0.05$ \\
$\mathrm{Co}_{34} \mathrm{Fe}_{8} \mathrm{Ag}_{54} \mathrm{Cu}_{4} 650^{\circ} \mathrm{C}$ & 0.33 & 575 & 2300 & 8700 & $0.30 \pm 0.05$ \\
$\mathrm{Co}_{34} \mathrm{Fe}_{8} \mathrm{Ag}_{54} \mathrm{Cu}_{4} 750^{\circ} \mathrm{C}$ & 0.33 & 575 & 2344 & 9850 & $0.78 \pm 0.10$ \\
\hline \hline
\end{tabular}



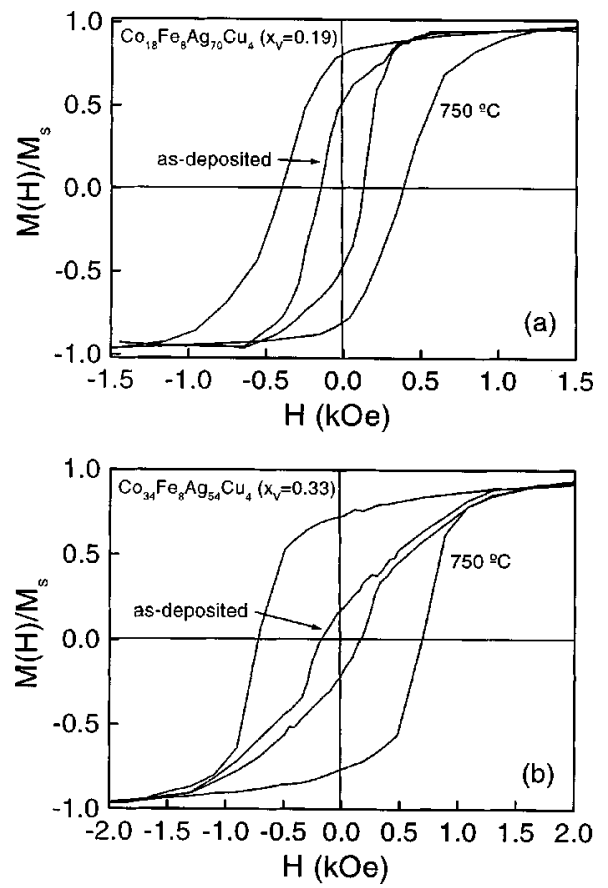

FIG. 2. Normalized parallel hysteresis loops at $5 \mathrm{~K}$ for as-deposited and $750{ }^{\circ} \mathrm{C}$ annealed samples: (a) $\mathrm{Co}_{18} \mathrm{Fe}_{8} \mathrm{Ag}_{70} \mathrm{Cu}_{4}\left(x_{v}=0.19\right)$ and (b) $\mathrm{Co}_{34} \mathrm{Fe}_{8} \mathrm{Ag}_{54} \mathrm{Cu}_{4}\left(x_{v}=0.33\right)$.

the synthesis conditions and not on the FM content. Beside, the degree of alloying was calculated using Vegard's law after carefully analyzing the shifting of the Bragg peaks, ${ }^{21}$ leading to a $2 \%$ of $\mathrm{CoFe}$ diluted in the $\mathrm{Ag}$ matrix for all as-deposited samples irrespective of $x_{v}$, indicating that the latter is $\mathrm{CoFe}$ saturated for all the concentrations studied.

\section{B. Temperature decay of the remanence and low field susceptibility}

Parallel hysteresis loops at $5 \mathrm{~K}$ are shown in Fig. 2 as a function of both the FM content and annealing temperature. It is evident from Fig. 2 that both $x_{v}$ and annealing temperature largely affect the $M_{r} / M_{s}$ ratio. The unusual shape of the hysteresis loop for the as-deposited samples with $x_{v} \geqslant 0.25$ is associated with an out-of-plane magnetic domain structure and is discussed in Ref. 27.

In as-deposited samples with $x_{v} \leqslant 0.25$, the perpendicular uniaxial anisotropy arising from the deformed fcc cell makes the in-plane $M_{r} / M_{s}$ ratio tend towards 0.5 at low temperature (Fig. 3). However, for some as-deposited samples, this value is greater than 0.5 , which might be due to the coexistence of axial distorted and cubic fcc crystals. In fact, strains are stronger nearer the substrate than further away from it (because of the stress relaxation associated with plastic deformations as the film grows). ${ }^{28}$ An axial distortion larger than $0.01 \%$ is at least needed to induce significant uniaxial anisotropy. ${ }^{24}$ Thus, for the $\mathrm{CoFe}-\mathrm{AgCu}$ as-deposited sample with $x_{v}=0.25$, the thickness is $355 \mathrm{~nm}$ and $M_{r} / M_{s}(5 \mathrm{~K})$ is about 0.6, while for the as-deposited sample with $x_{v}=0.19$, the thickness is $240 \mathrm{~nm}$ and $M_{r} / M_{s}(5 \mathrm{~K})$ is about 0.5 . The effect of thickness is largely responsible for the dispersion around 0.5 of the $M_{r} / M_{s}$ values at $x_{v}<0.25$ (Fig. 3).

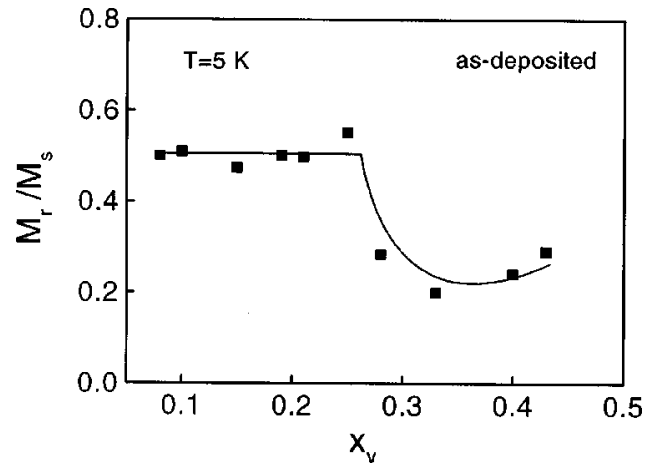

FIG. 3. $M_{r} / M_{s}$ ratio measured at $5 \mathrm{~K}$ as a function of the FM content, $x_{v}$, for $\mathrm{CoFe}-\mathrm{AgCu}$ as-deposited samples.

Figure 3 also shows that when $x_{v}>0.25$, the $M_{r} / M_{s}$ ratio decreases considerably (remanence breakdown). The dilution of $\mathrm{CoFe}$ atoms in the metallic matrix favors exchange interactions among FM particles ${ }^{5}$ either through the metallic matrix aided by the diluted paramagnetic atoms ${ }^{29}$ or because the matrix itself becomes a weak ferromagnet ${ }^{30}$ acting to couple the FM particles. Therefore, when magnetic particles are close to each other (at distances between particle surfaces smaller than about $1.5 \mathrm{~nm}$ at $x_{v} \geqslant 0.25$ ), interparticle interactions stabilize an out-of-plane stripe-like magnetic domain microstructure. 5,27 This result led us to conclude that magnetic percolation takes place well below the volume percolation threshold. Consequently, the remanent state after in-plane saturation displays an out-of-plane component of the magnetization, which does not contribute to the in-plane remanence. In all likelihood this effect is responsible for the remanence breakdown, leading to very small values of $M_{r} / M_{s}$ at low temperature. This is not the case for as-deposited samples with $x_{v}<0.25$, which do not show any out-of-plane component of the magnetization after in-plane saturation and display values of $M_{r} / M_{s} \sim 0.5$.

In addition to this remanence breakdown, the shape of the thermal dependence of the $M_{r} / M_{s}$ ratio for as-deposited samples significantly changes with $x_{v}$ (Fig. 4). The effects of interactions clearly favor low remanence at low temperatures and stabilize a blocked state at high temperature. Assuming that the mean blocking temperature, $\left\langle T_{B}\right\rangle$, may be calculated as $K V \sim 25 k_{B}\left\langle T_{B}\right\rangle$, where $V$ is the particle volume, $k_{B}$ the Boltzmann constant and $K \sim 0.35 \times 10^{6} \mathrm{erg} / \mathrm{cm}^{3}$, then CoFe particles of $3 \mathrm{~nm}$ in diameter should be superparamagnetic above about $10 \mathrm{~K}$. Thus, at room temperature, all asdeposited samples should be superparamagnetic and the $M_{r} / M_{s}$ ratio should be zero [Figs. 4(a)-4(c)]. However, in those samples with high FM content, magnetic correlations ensures that the $M_{r} / M_{s}$ ratio is kept almost constant with temperature, since these correlations prevent the thermal decay of $M_{r} / M_{s}$ [Fig. 4(d)].

Three curve types are observed when comparing the shape of the data shown in Fig. 4. The rapid decay with temperature of a weakly interacting distribution of fine magnetic particles is observed in as-deposited samples with $x_{v}$ $<0.19$, which evolves to a slower thermal decay as $x_{v}$ is progressively increased. For $x_{v}=0.10$ the curve corresponding to the annealing temperature at $750{ }^{\circ} \mathrm{C}$ is similar to that 

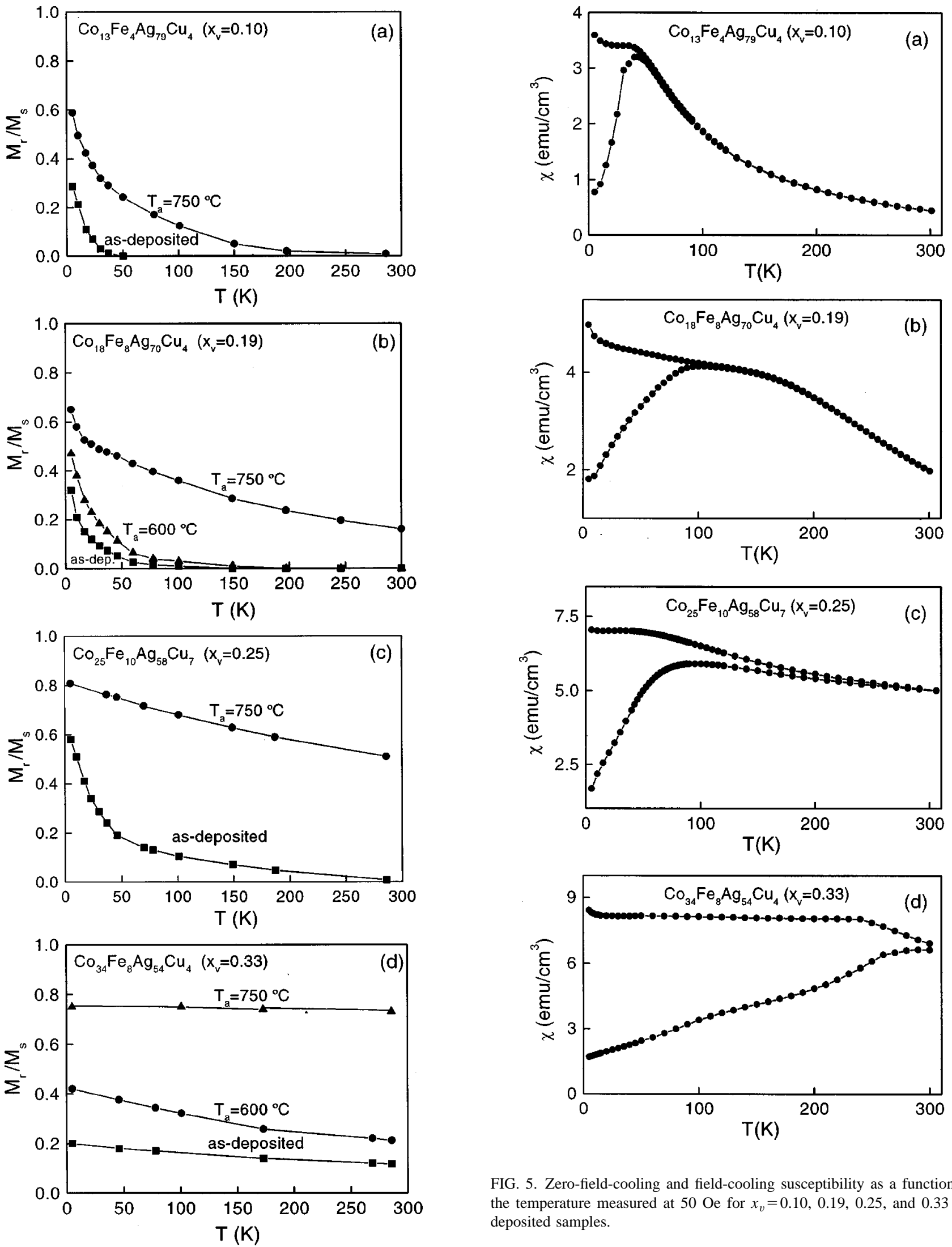

FIG. 5. Zero-field-cooling and field-cooling susceptibility as a function of the temperature measured at 50 Oe for $x_{v}=0.10,0.19,0.25$, and 0.33 asdeposited samples.

FIG. 4. $M_{r} / M_{s}$ ratio as a function of temperature for a variety of asdeposited and annealed $\mathrm{CoFe}-\mathrm{AgCu}$ samples: (a) $x_{v}=0.10$, (b) $x_{v}=0.19$, (c) $x_{v}=0.25$, and (d) $x_{v}=0.33$.

for the as-deposited sample, reflecting the slight evolution in the microstructure with annealing at low concentrations [Fig. 4(a)]. Correspondingly, the zero-field-cooled (ZFC) and field-cooled (FC) susceptibilities do not show a significant 
change with annealing [Fig. 5(a)] and display the typical features of a random distribution of weakly interacting fine magnetic particles. For $x_{v}=0.19$, the as-deposited and soft annealed samples also display a rapid decay in $M_{r} / M_{s}$ [Fig. 4(b)], while for the samples annealed at 650 and $750{ }^{\circ} \mathrm{C}$, a kind of bimodal behavior is observed corresponding to the superimposition of two contributions: One associated with the smallest particles (rapid decay) and the other associated with the largest particles and agglomerates (slow decay), due to the broad particle size distribution achieved with annealing in samples with $x_{v} \geqslant 0.19 .{ }^{21}$ This bimodal distribution in the annealed samples is confirmed through the thermal dependence of the ZFC and FC susceptibilities [see Figs. 5(b) and 6(a)]: The existence of small particles is evidenced by the fact that the ZFC curve decreases and the FC curve increases as temperature goes down; while the existence of large particles and aggregates is confirmed by the fact that the later thermal dependencies are rather smooth and no peak is observed in the ZFC curve. The as-deposited sample with $x_{v}=0.25$ shows similar behavior, with smaller $M_{r} / M_{s}$ values [Fig. 4(c)], while the annealed sample at $750{ }^{\circ} \mathrm{C}$ shows a quasilinear decay, which is due to the existence of very large particles and aggregates, in agreement with the very broad particle size distribution observed by transmission electron microscopy. ${ }^{21}$ In this case, the bimodal behavior observed in the as-prepared and soft annealed samples is attributed to the coexistence of strongly correlated and weakly interacting particles: (i) The ZFC and FC susceptibilities [Figs. 5(c) and 6(b)] are almost flat above the temperature of the maximum of the ZFC curve; the magnetic irreversibility extends up to very high temperatures and the FC curve is also flat below the maximum of the ZFC curve, thus suggesting the presence of strong interparticle interactions; (ii) the ZFC curve displays a maximum and largely decreases at low temperature. However, for the 650 and $750{ }^{\circ} \mathrm{C}$ annealed samples the overall behavior is quite similar to that observed for $x_{v}=0.19$, but with larger particles ${ }^{21}$ and interactions [the FC curve is flatter; Fig. 6(c)]. Finally, for $x_{v}=0.33 M_{r} / M_{s}$ for asdeposited and all annealed samples displays a weak thermal decay [Fig. 4(d)], as a consequence of the strong magnetic correlations in the as-deposited samples and large magnetic particles in the annealed ones. This is in agreement with the ZFC and FC curves: (i) Taking into account the fact that the size distribution is rather the same for all as-deposited samples irrespective of $x_{v},{ }^{21}$ the evolution shown in Fig. 5 suggests that the interparticle interactions largely increase with $x_{v}$; (ii) the curves corresponding to the annealed samples for $x_{v}=0.33$ indicate the existence of very large particles and magnetic interactions (probably between the particles within the same aggregate).

The $M_{r} / M_{s}$ ratio increases progressively with annealing, approaching 0.8 at low temperature (Fig. 7), as expected for cubic symmetry. ${ }^{6,23}$ Through annealing, the microstructure evolves to a higher degree of crystallinity: Phase segregation and strain relaxation take place, the crystal symmetry tends progressively to cubic and the magnetic anisotropy axis moves towards the $\langle 100\rangle$ direction of the cubic cell, forming an angle of $54 \pm 5^{\circ}$ with respect to the film normal (Table I). This remanence increase might also be partially
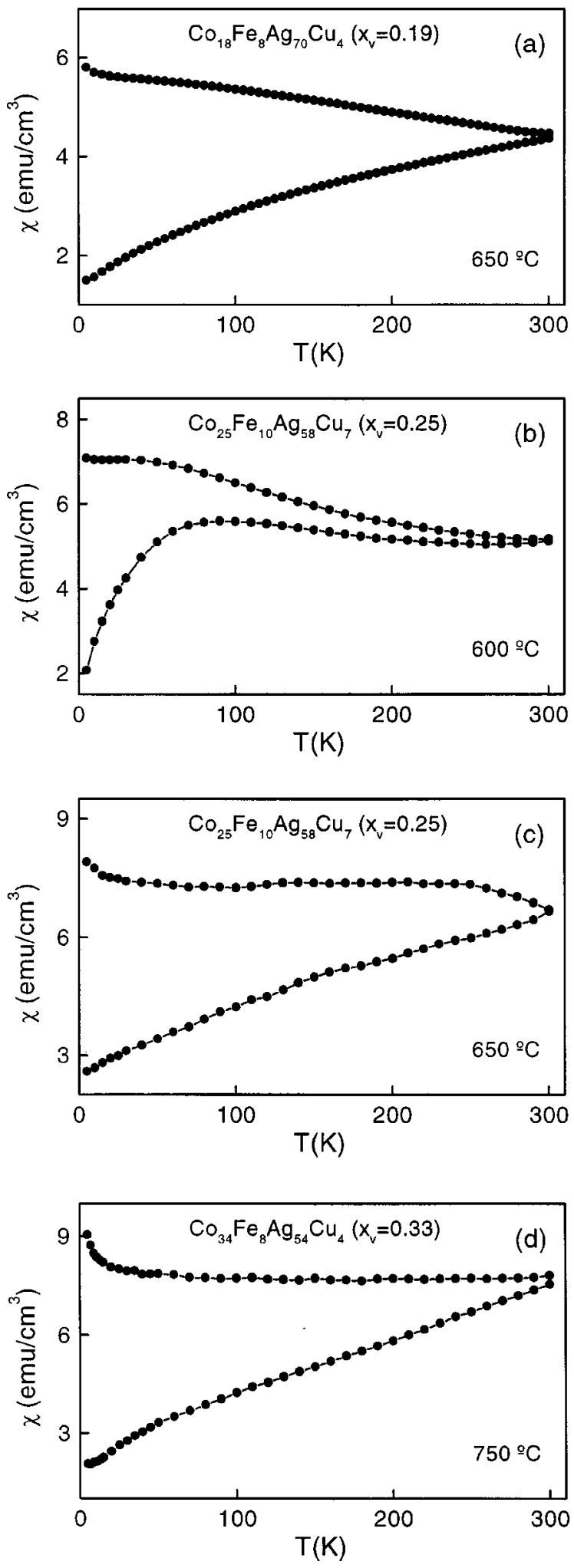

FIG. 6. Zero-field-cooling and field-cooling susceptibility as a function of the temperature measured at 50 Oe for the following annealed samples: (a) $x_{v}=0.19$ annealed at $650{ }^{\circ} \mathrm{C}$; (b) $x_{v}=0.25$, annealed at $600{ }^{\circ} \mathrm{C}$; (c) $x_{v}$ $=0.25$ annealed at $650{ }^{\circ} \mathrm{C}$; and (d) $x_{v}=0.33$ annealed at $750{ }^{\circ} \mathrm{C}$.

due to the random agglomeration of FM particles with annealing, which might withstand an enhanced remanence due to exchange interactions. Transmission electron microscopy showed that particles coalesce during annealing and twins, 


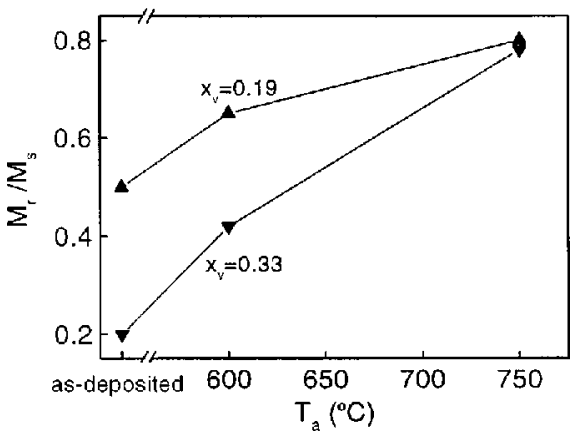

FIG. 7. $M_{r} / M_{s}$ ratio measured at $5 \mathrm{~K}$ as a function of the annealing temperature $\left(T_{a}\right)$ for two FM contents; $x_{v}=0.19$ and $x_{v}=0.33$.

transparencies and Moiré fringes were observed, suggesting the formation of large particle aggregates. ${ }^{21}$ However, we believe that it is the cubic symmetry that leads to an $M_{r} / M_{s}$ value of about 0.8 in highly annealed samples, since similar $M_{r} / M_{s}$ values are found for $x_{v}=0.19$ and $x_{v}=0.33$ (see Fig. 7). Even for $x_{v}=0.10$, where structural analysis showed almost no agglomeration, the $M_{r} / M_{s}$ value after annealing at $750{ }^{\circ} \mathrm{C}$ is close to 0.8 at low temperature [Fig. 4(a)].

For samples annealed at $750{ }^{\circ} \mathrm{C},\left\langle T_{B}\right\rangle$ should be well above room temperature, thus the weak thermal dependence of $M_{r} / M_{s}$ for the $750{ }^{\circ} \mathrm{C}$ annealed samples at high $x_{v}$ [Figs. 4(c) and 4(d)] has to be attributable to the large size of the FM particles rather than to interparticle interactions. In addition, as the $\mathrm{CoFe}$ spins diluted in the matrix are completely segregated and the perpendicular anisotropy is removed, no out-of-plane component of the magnetization is observed in the annealed samples, ${ }^{5,27}$ suggesting the formation of large in-plane domains. Consequently, no reduction in the remanence is observed.

The interpretation given above for the thermal dependence of $M_{r} / M_{s}$ as a function of $x_{v}$ and annealing temperature is reinforced through the analysis of the magnetic field dependence of the so-called $\Delta M$ plots (Fig. 8), which are based in the comparison of the isothermal remanent magnetization and the demagnetizing remanence curves (see Ref. 31). $\Delta M$ plot is a powerful method to determine the strength and nature of the macroscopic dominant interaction in the sample. As-deposited samples below the physical percolation threshold display negative $\Delta M$ values [Fig. 8(a)]. At low concentrations, this is attributed to the demagnetizing effect of the dipolar interactions among a random distribution of well separated particles. At $x_{v} \geqslant 0.25, \Delta M<0$ is attributed to the flux closure between antiparallel neighboring domains. Annealing causes particle growth and clusterization, leading to $\Delta M>0$ values at high $x_{v}$ [Fig. $\left.8(\mathrm{~b})\right]$. This fact is due to the increase in the direct exchange through the surface of neighboring particles in the same aggregate, at expense of dipolar interactions, which decreases as the clusters become more separated.

\section{CONCLUSIONS}

The $M_{r} / M_{s}$ ratio in magnetic granular alloys as a function of the concentration, temperature, and annealing has been studied. This ratio appears to be intricately linked to
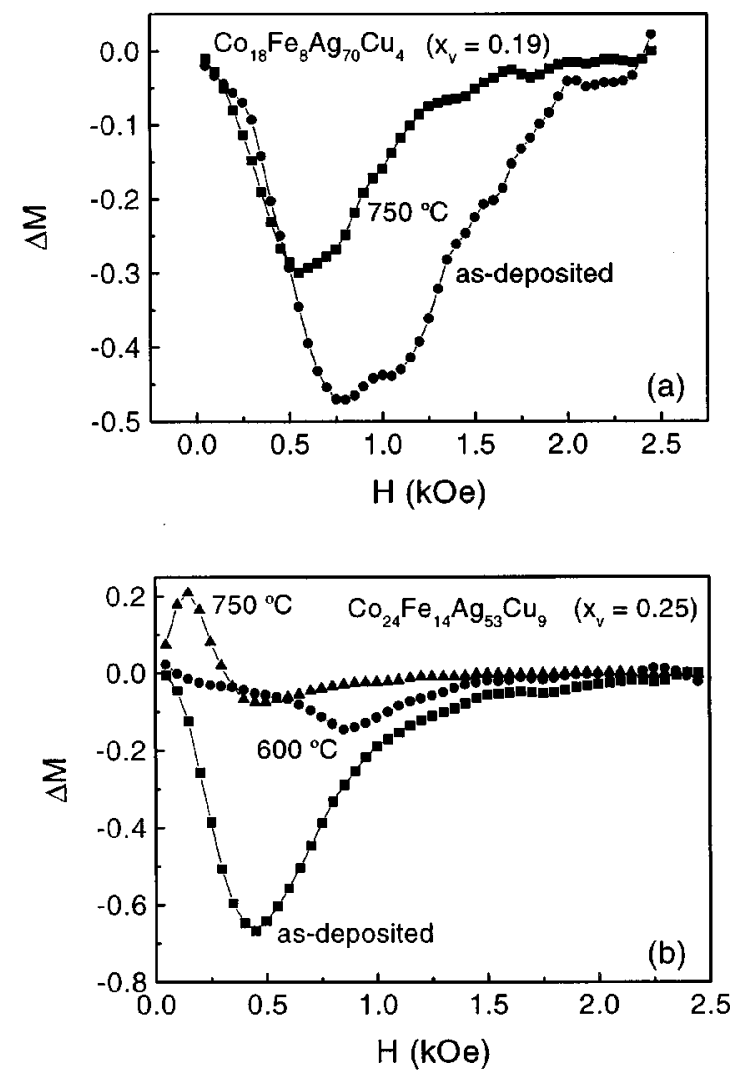

FIG. 8. $\Delta M$ plots for a variety of as-deposited and annealed samples at $5 \mathrm{~K}$ : (a) $x_{v}=0.19$; (b) $x_{v}=0.25$.

structural features, including residual stresses and axial distortions as small as $1 \%$. Moreover, while remanent enhancement has been widely studied in thin films and magnetic recording media, we have shown how remanence breakdown takes place in granular systems due to the stabilization of a longrange out-of-plane domain microstructure. Similar studies in $\mathrm{CoFe}-\mathrm{Cu}$ samples ${ }^{26}$ prepared under the same conditions also showed the evolution of $M_{r} / M_{s}$ from 0.5 in asdeposited samples to 0.8 in annealed ones, although no remanence breakdown was observed in as-deposited samples with high $x_{v}$. No out-of-plane component of the magnetization was observed after in-plane saturation, in agreement with the smaller anisotropy values and lower crystal texture displayed by these samples ${ }^{26}$ than those used here. It has also been shown that magnetic correlations are able to maintain a constant $M_{r} / M_{s}$ ratio up to room temperature, since they are able to avoid thermal decay of remanence, even for particles no more than $3 \mathrm{~nm}$ in size. These findings illustrate the extent to which the magnetic properties of granular alloys depend on the interplay between anisotropy, exchange, and dipolar interactions, which in turn depend crucially on the microstructure.

\section{ACKNOWLEDGMENTS}

Financial support from both the Spanish CICYT through the MAT-97-0404 and MAT-96-1642E projects and the Catalonian CIRIT through the 1998SGR0048 project are 
gratefully recognized. We are indebted to Professor A. Berkowitz (University of California at San Diego) for fruitful discussions.

${ }^{1}$ A. E. Berkowitz, J. R. Michell, M. J. Carey, A. P. Young, S. Zhang, F. E. Spada, F. T. Parker, H. Hutten, and G. Thomas, Phys. Rev. Lett. 68, 3745 (1992); J. Q. Xiao, J. S. Jiang, and C. Chien, ibid. 68, 3749 (1992).

${ }^{2}$ J. L. Dormann, D. Fiorani, and E. Tronc, Adv. Chem. Phys. 98, 283 (1997).

${ }^{3}$ R. W. Chantrell, in Science and Technology of Nanostructured Magnetic Materials, edited by G. C. Hadjipanayis and G. A. Prinz (Plenum, New York, 1991), NATO Advanced Series 259.

${ }^{4}$ See for example, J. L. Dormann and D. Fiorani, Magnetic Properties of Fine Particles (North-Holland, Amsterdam, 1992).

${ }^{5}$ V. Franco, X. Batlle, and A. Labarta, Eur. Phys. J. B (to be published); V. Franco, X. Batlle, A. Labarta, A. Valencia, F. Sandiumenge, M. L. Watson, and K. O'Grady, IEEE Trans. Magn. 34, 912 (1998).

${ }^{6}$ E. C. Stoner and E. P. Wohlfarth, Philos. Trans. R. Soc. London, Ser. A 240, 599 (1948).

${ }^{7}$ D. Kechrakos and K. Trohidou, Phys. Rev. B 58, 12169 (1998).

${ }^{8}$ M. El-Hilo, R. W. Chantrell, and K. O’Grady, J. Appl. Phys. 84, 5114 (1998).

${ }^{9}$ W. Luo, S. R. Nagel, T. F. Rosenbaum, and R. E. Rosensweig, Phys. Rev. Lett. 67, 2721 (1991).

${ }^{10}$ J. L. Dormann, R. Cherkaoui, L. Spinu, M. Noguès, F. Lucari, F. D’Orazio, D. Fiorani, A. Garcia, E. Tronc, and J. P. Jolivet, J. Magn. Magn. Mater. 187, L139 (1998).

${ }^{11}$ S. Morup, F. Bodker, P. V. Hendriksen, and S. Linderoth, Phys. Rev. B 52, 287 (1995).

${ }^{12}$ N. S. Walmsley, C. Dean, A. Hart, D. A. Parker, and R. W. Chantrell, J. Magn. Magn. Mater. 170, 81 (1997).
${ }^{13}$ G. F. Hughes, J. Appl. Phys. 54, 5306 (1983).

${ }^{14}$ M. El-Hilo, R. W. Chantell, and K. O'Grady, J. Appl. Phys. 81, 5582 (1997).

${ }^{15}$ S. M. Parhofer, J. Wecker, C. Kuhrt, G. Gieres, and L. Schultz, IEEE Trans. Magn. 32, 4437 (1996).

${ }^{16}$ L. Weil, J. Phys. Radium 12, 437 (1951).

${ }^{17}$ F. Soffge and E. Schmidbauer, J. Magn. Magn. Mater. 24, 54 (1981).

${ }^{18}$ S. Mahmood and I. Abu-Aljarayesh, J. Magn. Magn. Mater. 118, 193 (1993).

${ }^{19}$ M. El-Hilo, K. O'Grady, and R. W. Chantrell, J. Magn. Magn. Mater. 114, 295 (1992).

${ }^{20}$ S. R. Teixeira, B. Dieny, A. Chamberod, C. Cowache, S. Auffret, P. Auric, J. L. Rouviere, O. Redon, and J. Pierre, J. Phys.: Condens. Matter 6, 5545 (1994).

${ }^{21}$ V. Franco, X. Batlle, A. Labarta, J. Bassas, and F. Sandiumenge, Acta Mater. 47, 1661 (1999).

${ }^{22}$ B. Abeles, Applied Solid State Science: Advances in Materials and Device Research, edited by R. Wolfe (Academic, New York, 1976).

${ }^{23}$ S. Chikazumi, Physics of Magnetism (Krieger, Malabar, 1978).

${ }^{24}$ C. H. Lee, Hui He, F. J. Lamelas, W. Vavra, C. Uher, and R. Clarke, Phys. Rev. B 42, 1066 (1990).

${ }^{25}$ J. Q. Xiao, C. L. Chien, and A. Gavrin, J. Appl. Phys. 79, 5309 (1997).

${ }^{26}$ V. Franco, X. Batlle, and A. Labarta, J. Appl. Phys. 85, 7328 (1999).

${ }^{27}$ X. Batlle, V. Franco, A. Labarta, M. L. Watson, and K. O'Grady, Appl. Phys. Lett. 70, 132 (1997).

${ }^{28}$ J. H. Van der Merwe, J. Appl. Phys. 34, 117 (1963).

${ }^{29}$ A. Frydman and R. C. Dynes, Solid State Commun. 110, 485 (1999).

${ }^{30} 2 \%$ of $\mathrm{Fe}$ in Pt yields a ferromagnet with a Curie temperature of $100 \mathrm{~K}$; see, for example, Landolt-Bornstein, Magnetic Properties of Metals, edited by O. Madelung (Springer, Berlin, 1991), 19/III.

${ }^{31}$ V. Franco, X. Batlle, A. Labarta, and K. O'Grady, J. Phys. D: Appl. Phys. 33, 609 (2000). 